\title{
Sleep Deprived and Overwhelmed: Sleep Behaviors of Medical Students in the USA
}

\author{
Frederick Grady ${ }^{1}$ - Laura Weiss Roberts ${ }^{2}$
}

Received: 17 August 2017 / Accepted: 28 August 2017 /Published online: 14 September 2017

(C) Academic Psychiatry 2017

Good sleep is essential to good health. Good sleep strengthens cognitive capacities, such as memory and learning, and reduces the likelihood of making mistakes [1, 2]. For these reasons, the sleeping habits of medical students are important to understand. In this issue of Academic Psychiatry, three valuable empirical papers spontaneously submitted to the journal address this neglected topic in undergraduate medical education. Two studies were conducted at individual medical schools on the two US coasts and the other study involved 860 medical students from 49 different US medical colleges. Taken together, this unique set of data-based papers tells us a great deal about the current state of sleep among our early career colleagues.

\section{Sleep Behaviors of Medical Students}

Ahmed et al. [3] performed a cross-sectional study of medical students at SUNY Downstate Medical School $(n=261)$. The authors found that, on average, more than $70 \%$ of students felt they needed $7 \mathrm{~h}$ of sleep each night; however, only one quarter of students slept an average of $7 \mathrm{~h}$ nightly. Most students slept considerably fewer hours routinely, and during the week of an examination, only $15 \%$ of preclinical students and $19 \%$ of clinical students had more than $7 \mathrm{~h}$ of sleep [3]. Clinical students had considerably more sleepiness than preclinical students and clinical students were also more knowledgeable

Laura Weiss Roberts

lwroberts.author@gmail.com

1 Meharry Medical College, Nashville, TN, USA

2 Stanford University, Stanford, CA, USA about the topic of sleep. Preclinical and clinical students held similar views about sleep, as measured by the Dysfunctional Beliefs and Attitudes about Sleep (DBAS) scale [3]. This study also examined whether students used prescription sleep medication, over-the-counter sleeping pills, or behavioral sleep strategies. Most students reported that they "never" used prescription medications $(80 \%)$ or over-the-counter sleeping pills $(67 \%)$, while a small number reported using these methods "often/daily" (2\%, $7 \%$, respectively). Behavioral strategies were endorsed more commonly, with $13 \%$ using these approaches "often/daily" and 14\% using them "occasionally/monthly." Overall, clinical students were more likely than preclinical students to use prescription medication, overthe-counter sleeping pills, and behavioral strategies.

In their cross-sectional online survey of medical students $(n=860)$ across 49 medical schools in 29 states, Ayala et al. [4] similarly found that students slept less than $7 \mathrm{~h}$ each night (6.83 $\mathrm{h}$, on average over the prior 4 weeks), with students receiving the least sleep in year 3 and the most sleep in year 2 of medical school. Students in their last year had fewer sleep problems, as measured by the Sleep Problems Index, which is a subset of items from the well-validated Medical Outcomes Sleep Study Scale.

A vital and unique focus of the analyses in Ayala et al.'s study [4] related to the diminished quality of sleep of minority students. Minority students, defined as non-Caucasian, had more somnolence, which is reflective of daytime sleepiness, as well as lower self-reported levels of sleep adequacy and fewer hours of sleep per night. Minority students had more sleep difficulties on the Sleep Problems Index as well. In light of the increased levels of depression and anxiety among minority medical students [5] and the relationship between poor sleep, psychiatric disorders, and thoughts of self-harm [6, 7], these sleep-related findings are very important and warrant further empirical inquiry. 
Johnson et al. [8] examined two consecutive cohorts of second year medical students and performed analyses to better understand amount of sleep, daytime sleepiness, hazardous driving, and quality of life among these physicians-in-training. Roughly one quarter of students reported that they were receiving an "ideal" amount of sleep. Students with less sleep had more daytime sleepiness, as measured by the Epworth Sleepiness Scale, were more likely to report "nodding off" while driving during the academic year, felt more overwhelmed and "down," and expressed greater dissatisfaction with their quality of life on a number of measures. One in 11 students stated that they had dozed off while driving, and this pattern was greater among students who had slept less than $6 \mathrm{~h}$ the night before [8]. Low sleep quantity was also correlated with depression and burnout. Interestingly, amount of sleep was not associated with self-perceived ability to retain information, efficiency of study, or attendance at non-required classes. Although their project had incorporated the responses of second year students only, the authors asserted that their findings related to preclinical students in general. Future studies can further clarify whether the sleeping habits of first year and second year medical students are, in fact, similar.

These three studies suggest that the majority of medical students may be lacking in sufficient sleep. These studies have limitations, such as their reliance on self-report and low response rates, and, for these reasons, their generalizability remains uncertain. One of the studies focused only on second year medical students. The studies did not explore important topics such as curricular attention to sleep topics, knowledge of behavioral health and self-management health principles, perceived stressors, the role of mistreatment in medical school, the contribution of substance use or abuse (e.g., stimulants), and the personal health habits of medical students, e.g., use of caffeinated beverages or nicotine products, use of short naps, exercise routines, and adequacy of nutrition. These studies also did not examine considerations such as on-call schedules. Beyond sleep deprivation, these studies suggest that at least some medical students are feeling overwhelmed, which may be a cause, effect, correlation of sleep deprivation, or all of the above. The possible connections across these aspects of medical students' lives offer compelling empirical questions, which are important not only for students' well-being, but also for the well-being of current and future patients.

To highlight this last point, a key topic for future study is the relationship between sleep, alertness, and the making of mistakes, including the problem of drowsy driving. With respect to mistakes, the third leading cause of death in the USA is, unfortunately, medical errors [9]. Error rates are higher in the USA than in other countries such as Canada, Australia, New Zealand, Germany, and the UK [9]. Medical errors have been associated with fatigue and with physician "burnout" [10]. Medical students may be putting lifetime habits in place during their training; thus, interventions to strengthen their sleep behaviors may have enduring benefits for the health of the workforce and the health of the public. In addition, strengthening sleep habits may lessen the likelihood of driving while fatigued, which is a serious public hazard. Fatigued driving has been shown to be similar to driving while under the influence of alcohol in terms of safety consequences [11]. Despite this, over half of US adults have stated that they have driven while being drowsy [11]. According to the CDC, 1 in every 25 adult drivers (aged 18 years or older) has reported falling asleep while driving [12]. In 2013, drowsy drivers were responsible for 72,000 crashes, 44,000 injuries, and 800 deaths [12]. The extent to which physicians-in-training have been responsible for injuries or deaths related to drowsy driving is unknown and is another topic worthy of study.

Although there is clearly a need to address deeper issues regarding students' sleep, these studies represent an advance in the field of medical education. They underscore the value of further investigating the sleep behaviors of physicians-intraining, particularly the sleep behaviors of certain subsets of students, specifically undergraduate medical students. Funding for such investigations should be a priority, given the repercussions in the workforce and in public health. Such work will allow us to better assess the seriousness of sleep problems among physicians-in-training and physicians, the repercussions of these issues, and the potential targets for intervention.

\section{Advancing Sleep in Academic Psychiatry}

The field of academic psychiatry can do much to address the issue of sleep in undergraduate medical education and the problem of sleep deprivation among medical students. Stronger efforts are warranted to ensure adequate curricular attention to the topic of sleep as an important restorative brain function. The importance of sleep for undergraduate medical students is a neglected subject in medical school, along with other important health domains such as nutrition, sexual health, and behavioral health, as well as health prevention and promotion, and positive psychology and psychiatry.

Studying the behavioral aspect of sleep may be of great enduring value for future self-care and the care of patients. Rather than usual didactics, students would likely benefit from more intensive and focused educational and self-reflective activities, such as a sleep workshop, that would allow students to analyze their own sleeping behaviors and learn ways in which their sleeping behaviors could improve. Such a curricular offering might occur as early as first year orientation. Some medical schools may wish to provide the option of a quick professional evaluation to help those that may be suffering from sleep disorders. Such initiatives may offer students the opportunity to identify and address their poor sleep quality, 
which may improve their ability to perform in school. Faculty in psychiatry might also use their expertise to consult with members of curriculum committees to consider whether curricular planning could be tailored to help with students' sleep, e.g., the timing of examinations or the time of day in which mandatory classes are offered. We also suggest that faculty members and residents in academic departments of psychiatry do all they can to mentor and serve as good role models for students in relation to the topic of sleep as a key element of self-care.

The study of sleep behaviors of both physicians-intraining and health professionals more broadly should be a priority for researchers in academic medicine and in academic psychiatry in particular. Understanding sleep is clearly valuable to understanding the well-being and mental health of physicians and will help to ensure the safety of the workforce and the health of the public, as the three studies here suggest.

Academic psychiatry should also advocate for the field of Sleep Medicine and for the importance of studying the role of sleep in mental health and well-being. Sleep Medicine is an interdisciplinary field that has become a focus of pulmonary specialists in many medical schools, but is still relatively neglected by academic psychiatrists, despite evidence that behavioral interventions for sleep disorders are greatly beneficial to patients. More in-depth empirical investigation of sleep behaviors among children, adolescents, adults, and elders is needed, especially in relation to mental disorders. Academic psychiatrists and psychologists should support the advancement of Sleep Medicine.

In conclusion, this collection of reports from three empirical studies underscores the importance of sleep in relation to medical student health and well-being. These papers came to the journal spontaneously and were not part of an invited call for papers. From this observation, we conclude that there is growing recognition of the central role of sleep in ensuring the health of physicians-in-training as well as minimizing the harms associated with sleep deprivation. We hope that the empirical study of sleep behaviors of medical students continues and deepens and that this effort leads to more robust sleep curricula and more attuned sleep behavior interventions for physicians-in-training. Such efforts will do much to protect and strengthen the well-being of our early career colleagues and those entrusted to their care.

\section{Compliance with Ethical Standards}

Disclosure On behalf of all authors, the corresponding author states that there is no conflict of interest.

\section{References}

1. Watson NF, Badr MS, Belenky G, Bliwise DL, Buxton OM, et al. Recommended amount of sleep for a healthy adult: a joint consensus statement of the American Academy of Sleep Medicine and Sleep Research Society. J Clin Sleep Med. 2015;11:591-2.

2. Potkin KT, Bunney WE. Sleep improves memory: the effect of sleep on long term memory in early adolescence. PLoS One. 2012;7:e42191.

3. Ahmed N, Sadat M, Cukor D. Sleep knowledge and behaviors in medical students: results of a single center survey. Acad Psychiatry. 2017; https://doi.org/10.1007/s40596-016-0655-3.

4. Ayala EE, Berry R, Winseman JS, Mason HR. A cross-sectional snapshot of sleep quality and quantity among US medical students. Acad Psychiatry. 2017; https://doi.org/10.1007/s40596-016-0653-5.

5. Mental well-being in first year medical students: a comparison by race and gender: a report from the medical student CHANGE study. J Racial Ethn Health Disparities. 2015;2:403-13.

6. Pigeon WR, Pinquart M, Conner K. Meta-analysis of sleep disturbance and suicidal thoughts and behaviors. J Clin Psychiatry. 2012;73(9):e1160-7.

7. Bertolote JM, Fleishmann A. Suicide and psychiatric diagnosis: a worldwide perspective. World Psychiatry. 2002;1(3):181-5.

8. Johnson KM, Simon N, Wicks M, Barr K, O’Connor K, Schaad D. Amount of sleep, daytime sleepiness, hazardous driving, and quality of life of second year medical students. Acad Psychiatry. 2017; https://doi.org/10.1007/s40596-017-0668-6.

9. Anderson JG, Abrahamson K. Your health care may kill you: medical errors. Stud Health Technol Inform. 2017;234:13-7.

10. Kalmbach DA, Arnedt JT, Song PX, Guille C, Sen S. Sleep disturbance and short sleep as risk factors for depression and perceived medical errors in first-year residents. Sleep. 2017;40:zsw073.

11. National Sleep Foundation. "Drowsy driving vs. drunk driving: how similar are they?" 2017. Available from https:// sleepfoundation.org/sleep-topics/drowsy-driving-vs-drunkdriving-how-similar-are-they. Accessed 14 Aug.

12. Office of the Associate Director for Communication, Digital Media Branch, Division of Public Affairs. Drowsy driving: asleep at the wheel. Centers for Disease Control and Prevention. 2015. Available at https://www.cdc.gov/features/dsdrowsydriving/index.html. Accessed 9 Sept 2017. 